\title{
Seeing versus Knowing: The Temporal Dynamics of Real and Implied Colour Processing in the Human Brain
}

Abbreviated title: Real and Implied Colour Processing in the Brain

\section{Lina Teichmann ${ }^{*_{12}}$, Tijl Grootswagers ${ }^{1,23}$, Thomas Carlson ${ }^{23}$, Anina N. Rich ${ }^{1,24}$}

1l Perception in Action Research Centre \& Department of Cognitive Science, Macquarie University, Sydney, Australia

2I ARC Centre of Excellence in Cognition \& its Disorders, Macquarie University, Sydney, Australia

3I School of Psychology, University of Sydney, Australia

4l Centre for Elite Performance, Training and Expertise, Macquarie University, Sydney, Australia

Corresponding author's email: lina.teichmann@mq.edu.au

\section{ACKNOWLEDGEMENTS:}

This research was supported by the Australian Research Council (ARC) Centre of Excellence in Cognition and its Disorders, International Macquarie University Research Training Program Scholarships to LT \& TG, an ARC Future Fellowship (FT120100816) and ARC Discovery project (DP160101300) to TC and an ARC Discovery project (DP170101840) to ANR. 


\section{Abstract}

Colour is a defining feature of many objects, playing a crucial role in our ability to

3 rapidly recognise things in the world around us and make categorical distinctions. For example,

4 colour is a useful cue when distinguishing lemons from limes or blackberries from raspberries.

5 That means our representation of many objects includes key colour-related information. The

6 question addressed here is whether the neural representation activated by knowing that

7 something is red is the same as that activated when we actually see something red, particularly

8 in regard to timing. We addressed this question using neural timeseries

9 (magnetoencephalography, MEG) data to contrast real colour perception and implied object

10 colour activation. We applied multivariate pattern analysis (MVPA) to analyse the brain

11 activation patterns evoked by colour accessed via real colour perception and implied colour

12 activation. Applying MVPA to MEG data allows us here to focus on the temporal dynamics of

13 these processes. Male and female human participants $(\mathrm{N}=18)$ viewed isoluminant red and green

14 shapes and grey-scale, luminance-matched pictures of fruits and vegetables that are red (e.g.,

15 tomato) or green (e.g., kiwifruit) in nature. We show that the brain activation pattern evoked

16 by real colour perception is similar to implied colour activation, but that this pattern is

17 instantiated at a later time. These results suggest that a common colour representation can be

18 triggered by activating object representations from memory and perceiving colours. 


\section{Introduction}

Throughout our lives, we learn statistical regularities about objects in our environment.

We acquire knowledge about their typical perceptual features, which motor actions are required

to interact with them, and in which context they usually appear. For example, we know that a

tomato is round and red, we can eat it and it appears in the wider context of food. Our neural representations of objects therefore need to encompass a conceptual combination of these learnt

attributes spanning from perception to action and semantic knowledge (A. Martin, Haxby,

Lalonde, Wiggs, \& Ungerleider, 1995). The activation of object representations is likely to involve a widespread, distributed activation of several brain regions (Patterson, Nestor, \& Rogers, 2007) with some brain areas responding preferentially to object colour (e.g., Seymour, Williams, \& Rich, 2015). Several neuroimaging studies have compared perceiving colour and accessing object-colour knowledge from memory, finding evidence that similar brain areas are involved in these two processes (e.g., Bannert \& Bartels, 2013; A. Martin et al., 1995;

Vandenbroucke, Fahrenfort, Meuwese, Scholte, \& Lamme, 2014). Using magnetoencephalography (MEG), we look at the neural timecourse of 'real' (by which we mean 'induced by wavelengths of light') colour perception versus implied object-colour activation from memory.

Associations between objects and typical or implied colours are acquired through experience (Bartleson, 1960; Hering, 1920) and are activated effortlessly and involuntarily

40 (Bramão, Faísca, Petersson, \& Reis, 2010; Chiou \& Rich, 2014). The activation of objectcolour knowledge is part of the dynamic interaction between perceptual processes and activation of prior conceptual knowledge to evaluate sensory input (Collins \& Olson, 2014; Engel, Fries, \& Singer, 2001; Goldstone, de Leeuw, \& Landy, 2015). One of the central questions is how object-colour knowledge interacts or overlaps with colour representations generated by external stimuli. There is behavioural evidence that object-colour knowledge can 
influence colour perception. Hansen, Olkkonen, Walter, \& Gegenfurtner (2006) found that participants overcompensated for implied colours when they were asked to change the colour of colour-diagnostic objects to be achromatic. For example, a banana would be adjusted towards the blue side of grey, showing the influence of the implied colour yellow. Similarly, Witzel (2016) showed that participants selected an image of an object as achromatic more often when its colour was modified to be the opposite of its implied colour (e.g., a bluish-grey banana). These results suggest that colour perception can be influenced by previously learnt object-colour associations (see Firestone and Scholl, (2016) for debates about the extent to which activation of colour from memory is identical to colour perception). Brain-imaging data, recorded with functional magnetic resonance imaging (fMRI), suggest that brain activation corresponding to implied object colour activation shares characteristics with real colour perception: Retrieving the knowledge that a banana is yellow activates brain areas in or around the V4 complex, which is involved in colour perception (Bannert \& Bartels, 2013; Barsalou, Simmons, Barbey, \& Wilson, 2003; Chao \& Martin, 1999; Rich et al., 2006; Simmons et al., 2007; Vandenbroucke et al., 2014). This suggests that activation of implied colour rests on a similar neural architecture as real colour perception.

These results suggest that similar brain areas are active when perceiving colour and accessing implied colour, which may drive the behavioural interactions between the two (e.g., Hansen et al., 2006). Real colour activations occur very early in visual processing, whereas implied colour presumably is only activated once the object is processed at a higher level. Hence, there could be a temporal delay for activity driven by implied colour in comparison to activity driven by perceived colour. As the signal measured by fMRI is slow, it is not a suitable method to distinguish fine temporal differences between real and implied object colour processing. In the current study, we use multivariate pattern analysis (MVPA) on MEG data (Grootswagers, Wardle, \& Carlson, 2017) to compare the brain activation patterns evoked by 
colour perception and implied object colour activation. MEG has fine temporal resolution, and with MVPA we can detect patterns across the sensors at each time point that are reliable enough to train an algorithm to classify different categories of stimulus. Here, we use these methods to test whether a classifier trained on 'real colour' can successfully decode 'implied colour'. Such cross-generalisation can only occur if there is sufficient similarity in the neural signals. This approach enables us to contrast the temporal dynamics of real and implied colour processing, shedding light on the interaction between perceptual processing and activation of object representations.

\begin{abstract}
Methods
Participants. 20 healthy volunteers (12 female, mean age $=27.6$ years, $\mathrm{SD}=6.6$ years) completed the study. All participants reported normal or corrected-to-normal vision including normal colour vision. Participants gave informed consent before the experiment and were reimbursed with \$20/hour. During the MEG recording, participants were asked to complete a target-detection task to ensure they were attentive. Two participants performed more than three standard deviations below the group mean on this task, suggesting they did not pay attention to the stimuli, and were therefore excluded from analysis, leaving 18 participants in total. The study was approved by the Macquarie University Human Research Ethics Committee.
\end{abstract}

Procedure. While lying in the magnetically shielded room (MSR) for MEG recordings, participants first completed a colour flicker task (Kaiser, 1991) to equate the coloured stimuli in perceptual luminance. Then they completed the main target-detection task. We used only two colours to increase the power of our analysis. If there are luminance differences between the colour categories, the classifier can use this strong signal to discriminate the categories instead of relying on colour. While previous studies have shown the greatest behavioural effects for colours along the daylight axis (yellow-blue, Hansen et al., (2006)), these are not 
97 feasible colours for the current design: equiluminant blue and yellow stimuli no longer look clearly blue and yellow. We chose red and green as the two colours as they can be matched for luminance, and we included varying exemplars of these two hue categories to ensure any potential remaining slight differences in luminance could not be used by a classifier to

101 distinguish the colour categories.

Colour Flicker Task. In the colour flicker task, participants were presented with red and green circles ( $5 \times 5$ degrees visual angle) in the centre of the screen. The colours alternated at a rate of $30 \mathrm{~Hz}$. Participants completed 2 runs of 5 trials each. In each trial, one red-green combination was used. The red colour was kept consistent throughout each trial while participants were asked to use a button box to adjust the luminance level of green and report when they perceived the least amount of flickering. The HSV (hue, saturation, value) values for each green shade were then recorded. This procedure was repeated in the second run. The average HSV values between the two runs was then computed, yielding five shades of red and

110 green equated for perceptual luminance. Using different shades of red and green which were 111 each equated for perceptual luminance minimises the degree that any luminance difference 112 between the categories could influence the results (see Table 1 [supplementary materials] 113 summarising individual HSV values used).

Target-Detection Task. In the main target-detection task (Figure 1A), participants

115 completed eight blocks of 440 trials each. There were two different types of blocks: implied

116 colour and real colour. Block types alternated for each participant and the overall order was 117 counterbalanced across participants. In the implied colour blocks, participants viewed

118 luminance-equated (SHINE toolbox Willenbockel et al. (2010)) grey-scale images of colour

119 diagnostic objects (see Figure 1A). Equating the overall luminance ensures that differences in

120 MEG response patterns are not caused by luminance differences between the 'usually red' and 'usually green' categories. To increase variability in the stimulus set, half the stimuli depicted 
122 a single item on the screen (e.g., one strawberry) and the other half were multiple, partially

123 overlapping items (e.g., three strawberries). Having several different stimuli in each category

124 helps to minimise the influence of low-level features such as edges and shapes on the results.

125 In the real colour blocks, participants viewed five different abstract shapes. Each shape was

126 filled in one of the red and green shades which had been equated for perceptual luminance with

127 the colour flicker task. Each shape occurred equally often in red and green. To match the stimuli

128 presented in the implied colour block, half of the shapes were single shapes (e.g., one square)

129 on the screen while the other half consisted of partially overlapping shapes (e.g., three squares).

130 All stimuli (objects and shapes) contained the same number of pixels (Figure 1A).

131 In both block types, presentation location varied randomly by $\sim 1$ degree visual angle

132 around the central fixation cross. Changing the spatial location of the stimulus images adds

133 variability to the retinal image, again reducing the influence low-level visual features have on

134 the results. Participants were asked to press a button when they saw an image of the target

135 shape (cross) or target object (capsicum). Every block had 40 target trials. All target trials were

136 discarded before the MEG data analysis. On average, participants detected $98.51 \%(\mathrm{SD}=$

$137 \quad 0.013 \%)$ of target stimuli. 

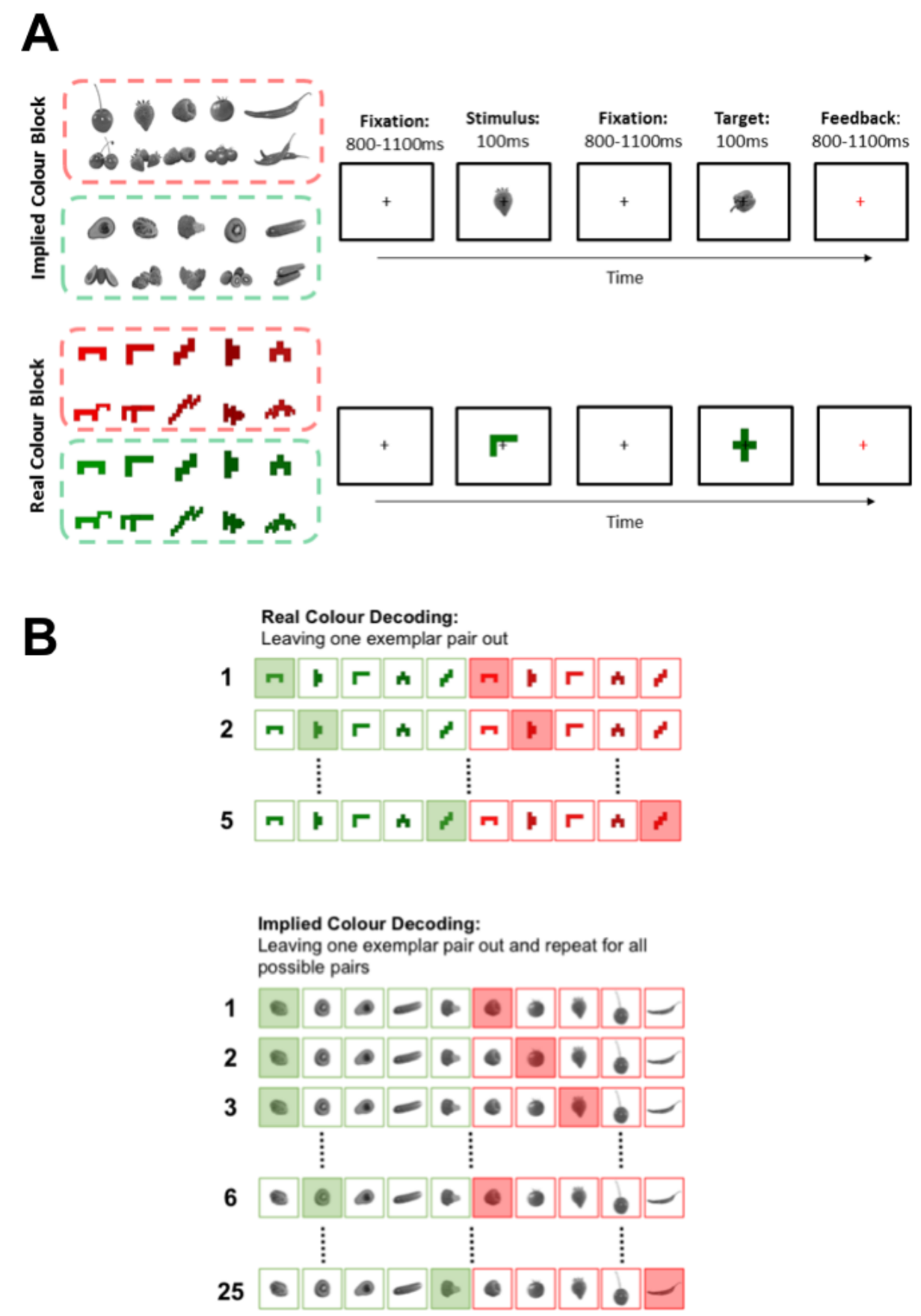

Figure 1: (A) Target-detection task and stimuli for both implied colour (top panel) and real colour (bottom panel) blocks. Participants were asked to press a button as soon as they saw the target (capsicum or cross). If they pressed the button after a target (correct detection) the fixation cross would turn green briefly, if they missed the target it would turn red. (B) Crossvalidation approach used for real (top) and implied (bottom) colour decoding analyses. Every row shows which trials were used for the training set (clear) and which trials were used for the testing set (shaded). Trials with the same exemplar are never in the training and the testing set. In the real colour decoding analysis, we split the data in 5 different ways, always leaving one pair of the same shape with matched luminance out. In the implied colour decoding analysis, we split the data in $\mathbf{2 5}$ different ways, leaving all possible exemplar pairs out once. The classification accuracy is an average of the classifier performance for each of these divisions. 
Apparatus and Pre-processing. The stimuli had a visual angle of approximately 5 x 5

140 degrees and were projected onto a translucent screen mounted in the magnetically shielded

141 room. The stimulus display system used was an Epsom projector (EB-G7400U) with a refresh

142 rate of $60 \mathrm{~Hz}$. The projector display size had approximately 10.02 x 19.9 degrees visual angle

143 and the resolution of the display was set to $1280 \times 720$ pixels. Stimuli were presented using

144 MATLAB with Psychtoolbox extension (Kleiner et al., 2007; Pelli, 1997). The neuromagnetic

145 recordings were obtained with a whole-head axial gradiometer MEG (KIT, Kanazawa, Japan),

146 containing 160 axial gradiometers. The frequency of recording was $1000 \mathrm{~Hz}$. FieldTrip

147 (Oostenveld, Fries, Maris, \& Schoffelen, 2011) was used to pre-process the data. We used a

148 low-pass filter of $200 \mathrm{~Hz}$ and a high-pass filter of $0.03 \mathrm{~Hz}$ online. Stimulus onsets were

149 determined with a photodiode that detected light change when a stimulus came on the screen.

150 Trials were epoched from -100 to $800 \mathrm{~ms}$ relative to stimulus onset and downsampled to $200 \mathrm{~Hz}$

151 (5ms resolution). All target trials were removed. We performed no further preprocessing steps

152 (e.g., channel selection, artefact correction), leaving our data in the rawest possible form. This

153 choice was motivated by recent work showing that traditional preprocessing choices can

154 introduce artefacts in the data that have a strong effect on multivariate analyses (van Driel,

155 Olivers, \& Fahrenfort, 2019).

156 Decoding Analysis. We conducted four separate decoding analyses using linear

157 discriminant classifiers (LDA) implemented in CoSMoMVPA (Oosterhof, Connolly, \&

158 Haxby, 2016). First, to test whether we can decode perception of red versus green, we analysed

159 the data from the real colour (shape) blocks. We tested whether we could decode the colour of

160 our abstract shapes for each person. The classifier was trained on distinguishing the activity

161 patterns evoked by red versus green shapes at each timepoint using $80 \%$ of the real colour data.

162 We then used the classifier to predict the colour of each stimulus at every timepoint in the remaining $20 \%$ of the real colour data. To divide the data into training and testing set, we used 
164 an independent exemplar cross-validation approach (Carlson, Tovar, Alink, \& Kriegeskorte,

165 2013), leaving out one exemplar pair with matched luminance (e.g., red and green L-shape,

166 matched for perceptual luminance). This process was repeated over all folds so that each

167 exemplar pair was in the training and the testing set once (5-fold cross-validation). Hence, the

168 colours in each fold were balanced (Figure 1B).

169 Second, to assess whether we can decode implied colour from grey-scale objects, we

trained a classifier to distinguish trials of grey-scale objects that are associated with red versus

green. As in the analysis described above, we used an independent cross-validation approach

and trained the classifier on $80 \%$ of the implied colour data and tested its performance on the

remaining $20 \%$ of implied colour data. Because the greyscale objects in the red and green

condition varied in more ways than just their implied colours, we left out both possible

to which visual features such as shape would be used by the classifier. We selected trials based

on label for both colour categories (e.g., all strawberry and kiwifruit trials). Note that there were two instances of each stimulus (e.g., an image of one strawberry and an image of three strawberries) and these were considered the same object for the leave-one-out procedure. We trained our classifier to distinguish between activity patterns evoked by all stimuli except the selected stimuli and tested its performance on the left-out trials. We repeated this process to have every possible combination of green and red objects used once as the testing set (25-fold cross-validation), and report the average classification performance over all these combinations other than implied colour driving the effect, we still have to be cautious with the interpretation as there may be overall low-level differences across all the red and green objects. This is unavoidable when using natural objects. 
Last, we conducted a cross-decoding analysis across the two different block types, training the classifier on all real colour trials and testing on all implied colour trials. This crossdecoding analysis is highly conservative as everything about the stimuli differs between real colour and object colour trials, the only potential link is the implied colour of the objects to the real colour of the abstract shapes. If there are any low-level differences in the real colour decoding other than chromaticity (e.g., overall luminance difference), this would only decrease the likelihood of finding significant cross-generalisation to the implied colour trials. In addition, any differences in between the greyscale objects cannot drive an effect in the crossdecoding analysis, as the classifier is trained to distinguish the real colour shapes which are the same in the red and the green condition. conducted a time-generalisation analysis (Carlson, Hogendoorn, Kanai, Mesik, \& Turret, 2011; King \& Dehaene, 2014), training the classifier at each timepoint on the real colour trials and then testing on each timepoint in implied colour trials, yielding an accuracy score for all pairs of training and testing timepoints. This technique allows us to test for similar activation patterns that do not occur at the same time.

Statistical Tests Classification Analyses. To assess whether the classifier could distinguish between red and green trials significantly above chance, we used random effects

207 Monte-Carlo cluster statistic (Maris \& Oostenveld, 2007) using Threshold Free Cluster

208 Enhancement (TFCE, Smith \& Nichols, 2009) as implemented in CoSMoMVPA (Oosterhof et al., 2016). The TFCE statistic represents the support from neighbouring time points, allowing

210 optimal detection of sharp peaks, as well as sustained weaker effects. First, a permutation test

211 was conducted by swapping the labels of complete trials and then we re-ran the analysis on the data with the shuffled labels. This was repeated 100 times per participant to generate subject- 
213 level null-distributions. Second, Monte-Carlo sampling was used to create a group-level null-

214 distribution consisting of 10,000 shuffled label permutations for the time-resolved decoding,

215 and 1000 for the time-generalisation analysis (to limit computation time). Third, these group-

216 level null-distributions were transformed into TFCE statistics (Smith \& Nichols, 2009). To

217 correct for multiple comparisons, we then selected the maximum TFCE value across time in

218 each of the null distributions. Finally, to assess whether decoding was above chance, we

219 transformed the true decoding values to TFCE statistics and compared them to the $95^{\text {th }}$

220 percentile of the corrected null distribution.

Behavioural data collection. In addition to our MEG experiment, we collected colour categorisation accuracies and reaction times on our stimuli from a new sample of 100 participants on Amazon's Mechanical Turk. Participants were presented with the red and green shapes and the grey-scale objects, each presented individually for $100 \mathrm{~ms}$, randomly intermingled. On the instructions screen, participants were told that they would see images that can be categorised as red or green. They were informed that some images would be shown in greyscale, but that these objects were typically associated with red or green. Their task was to categorise the images into these two categories as fast and as accurately as possible by responding with either "m" or " $\mathrm{z}$ " using a keyboard. This allowed us to first confirm that the objects we had selected were indeed typically associated with red or green, and second, to test whether there was a reaction time difference between real and implied colour categorisation.

232 Response-key mappings were randomly determined for each participant. Participants each completed 6 practice trials on objects that were not used in the experiment before the actual data collection began. Each participant was presented with each of the objects once. We calculated the mean accuracy and reaction times for the real and implied colour condition. 


\section{Results}

For our real colour decoding analysis, we trained the classifier to distinguish red from

green shapes and then tested whether it could distinguish red from green shapes in an independent set of data. The classifier was able to predict the colour above chance in a cluster stretching from 65 to $315 \mathrm{~ms}$, reflecting a signal modulated by colour (Figure 2, orange). show that the classifier can distinguish between the objects belonging to the red and green category significantly above chance in a cluster stretching from 190 to $215 \mathrm{~ms}$ and from 270 to $290 \mathrm{~ms}$ (Figure 2, blue). While this suggests that there is categorical difference between objects associated with red and green, the results of this particular analysis could be driven by an overall difference in object characteristics other than colour (e.g., if red objects tend to have more round edges than green objects), and we therefore do not interpret this further.

Our key analysis to test whether there is a representational overlap of real and object colour processing depends on cross-decoding: training a classifier on real colour stimuli and testing on grey-scale objects that have implied colours. We trained the classifier to distinguish between the red and green shapes and tested its performance on the grey-scale objects to see whether direct cross-generalisation between real and implied object colour is possible. In this analysis, the classifier is trained on identical shapes that only vary in terms of colour. Hence, this is the most conservative way of testing whether there is a representational overlap between real and implied colours. The cross-colour decoding was not significant at any point in the timeseries (Figure 2, yellow). Accessing implied colour, however, presumably requires first

261 accessing the general concept of the object. Therefore, real and implied colours may have a

262 similar representation but colour information could be accessed later when activated via objects 
in comparison to when colour is perceived. We therefore tested whether this is the case using red and green shapes at every timepoint and then tested whether it could cross-generalise to the grey-scale objects at any timepoint. The results of key cross-generalisation analyses are summarised in Figure 3, showing a cluster of significant cross-generalisation with a time-shift.

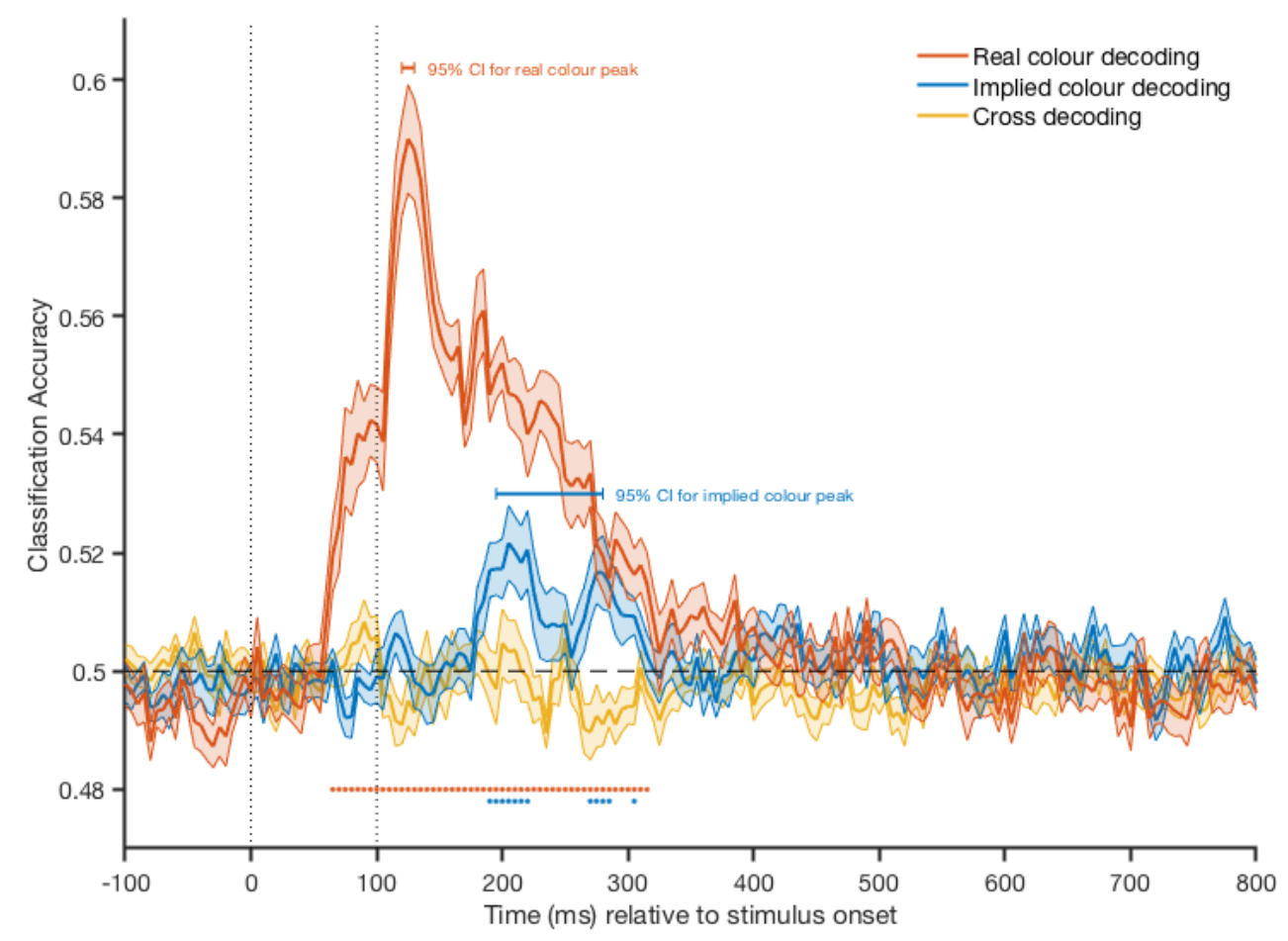

Figure 2: Classification accuracies for real colour (orange), implied colour (blue), and direct cross-colour (yellow) decoding over time. Vertical, dotted lines show stimulus onand offset. Dashed line indicates chance level (50\%). Shading indicates error bars around the mean (standard deviation of decoding accuracies across participants divided by the square root of the number of participants). Coloured dots depict significant timepoints corrected for multiple comparisons. The $95 \%$ confidence intervals for peak decoding latencies are plotted above the classification accuracies. 
270 The time-generalisation analysis revealed similar activation patterns between real and implied

271 colours when the classifier is trained on real colour at an earlier timepoint and tested on implied

272 colour at a later one (Figure 3A and 3B). These generalisation accuracies were statistically

273 above chance, even with our conservative correction for multiple comparisons (Figure 3C).

274 Inspecting the training timepoint with maximum decoding (140 ms) indicates that there is

275 above-chance decoding at later testing timepoints with peak decoding at $200 \mathrm{~ms}$ after stimulus

276 onset (Figure 3B). The results show that we can cross-decode between real and implied colours

277 when we train the classifier on real colours at timepoints between 140 to $160 \mathrm{~ms}$ and test it on

278 implied colours at a cluster from 200 to $215 \mathrm{~ms}$ (Figure 3C). Combining the off-diagonal shift

279 of the significant timepoints shows a median delay of $55 \mathrm{~ms}$ for implied colour testing times

280 compared to real colour training times (Figure 3D). Importantly, these results are unlikely to

281 be driven by anything else than colour as the classifier is trained on real colour trials in which

282 the only different stimulus characteristic was colour and tested on implied colour trials which

283 were achromatic. As a check, we also performed the reverse analysis (i.e., training the classifier

284 on implied colour trials and testing it on real colour trials) which showed the same results,

285 mirrored across the diagonal. The results highlight that there are similarities between real

286 colour and implied object colour patterns but this pattern is instantiated later for implied object

287 colours than for real colours. Note that above-chance cross-decoding does not mean we can

288 interpret that the processes involved in real and implied colour processing are identical.

289 However, the results show that there are sufficient similarities for the classifier to cross-

290 generalise from brain activation patterns evoked by perceiving red and green to brain activation

291 patterns evoked by viewing grey-scale images of objects that are associated with red and

292 green $^{1}$.

\footnotetext{
${ }^{1}$ Please note that these results are stable across different analysis parameters. For example, the effect remains when using a different classifier, a wider sliding time windows, and when averaging across trials in the training data, normalising the training data, and using principal component analysis.
} 

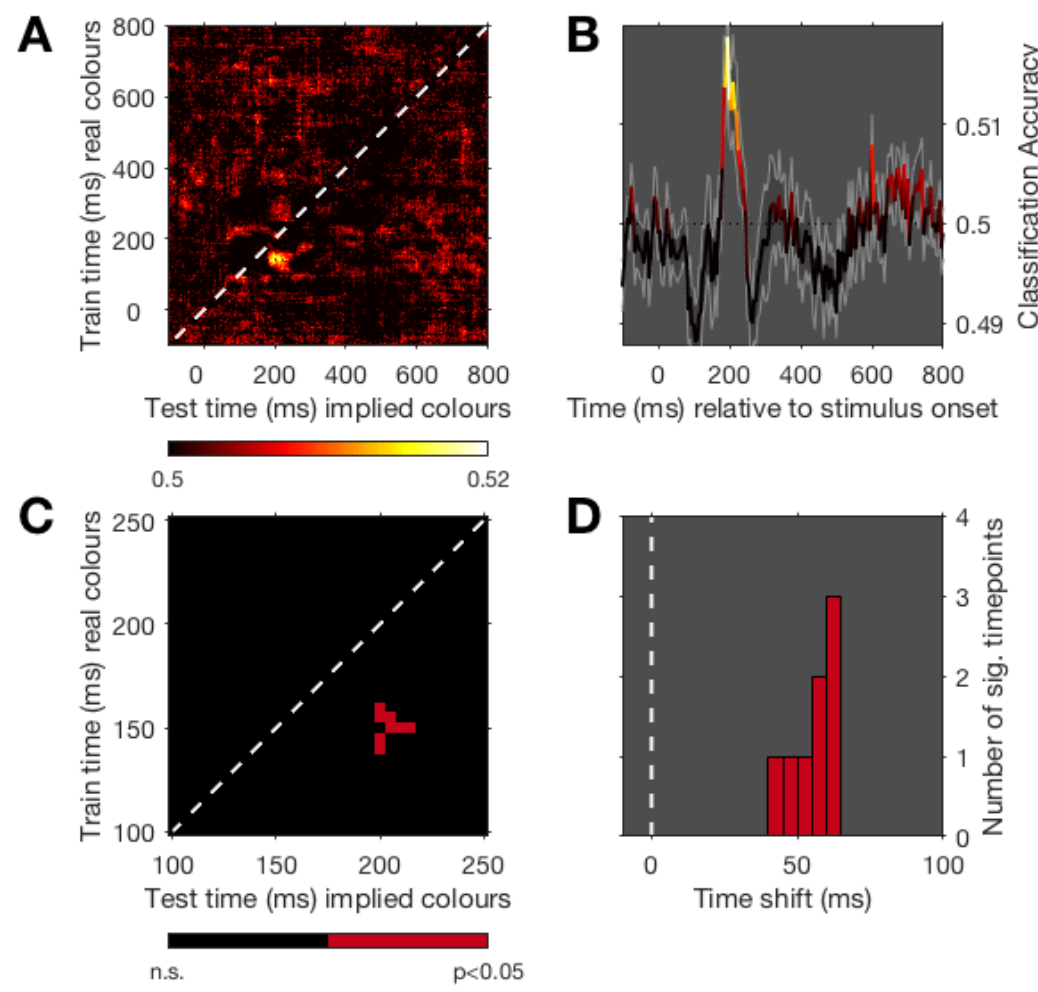

Figure 3: Results of the time-generalisation analysis. In this analysis, the classifier was trained on the real colour trials and tested on the implied colour trials. Panel A shows the classification accuracy at every possible train-test-time combination. Lighter colours indicate higher classification accuracy. Panel B shows the timecourse of the classification accuracy when the classifier relies on the training data at $140 \mathrm{~ms}$ (peak decoding at $200 \mathrm{~ms}$ ). Shading indicates error bar around the mean (standard deviation of decoding accuracies across participants divided by the square root of the number of participants). Panel $\mathrm{C}$ shows all training-testing-timepoint combinations where classification is significantly above chance (based on permutation, corrected for multiple comparisons). Note that the axes in $\mathrm{C}$ are different to show the significant timepoints. Panel D shows the time shift of all significant timepoints from the diagonal. The delay of colour representations activated via implied colour activation in comparison to real colour perception is $\sim 55 \mathrm{~ms}$.

These results predict that it takes more time to access implied colour than real colour,

presumably because one first has to access the concept of the object. We decided post-hoc to 
300 excluded all the incorrect responses and compared the correct reaction times to the real and

301 implied colour trials. Responding correctly to a real colour shape was on average $136 \mathrm{~ms}$ (SD

$302=85 \mathrm{~ms})$ faster than responding correctly to an implied colour of an object $(\mathrm{t}(95)=15.9, \mathrm{p}<0.05$, $30395 \%$ CI $[121.08,155.64])$. Real colour responses were also more accurate $(\mathrm{M}=91.5 \%, \mathrm{SD}=$ 304 0.08) than implied colour responses $(\mathrm{M}=80.4 \%, \mathrm{SD}=0.13)$. The accuracy scores for the real 305 and implied colour condition were significantly different $(\mathrm{t}(95)=8.07, \mathrm{p}<0.05,95 \%$ CI $[8.3$

306 13.8]). Using mTurk introduces variance to the experimental setup, including monitor settings

307 for colour, computer and internet speeds, all of which will increase the noise in the data; we do 308 not, therefore, interpret the specific difference in timing. Despite the variability, there is a clear 309 difference between the time taken for categorising colour in the two conditions. These results 310 are consistent with real colour perception being faster and easier than recalling implied colours, 311 in line with the prediction from our decoding results.

312 To test the relationship between the neural data and behavioural data further, we also 313 ran an exploratory analysis correlating the neural data of our sample with the behavioural data 314 of the independent set of mTurk participants. We correlated the stimulus-wise behavioural 315 categorisation data with the stimulus-wide MEG decoding accuracies for the implied colour 316 decoding analysis and examined how this correlation unfolds over time (Figure 4). The results 317 show that the neural data can be linked to the behavioural data from $\sim 200 \mathrm{~ms}$ after stimulus 318 onset which suggests that the information we decode can be used to generate behaviour (cf. de-

319 Wit, Alexander, Ekroll, \& Wagemans, 2016; Grootswagers, Cichy, \& Carlson, 2018; Williams, 320 Dang, \& Kanwisher, 2007). 
A

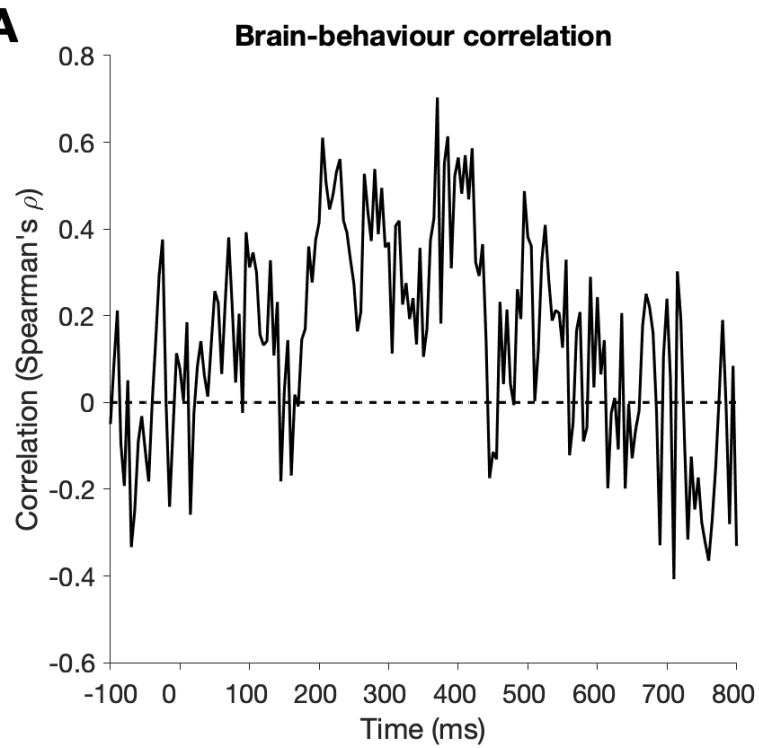

B

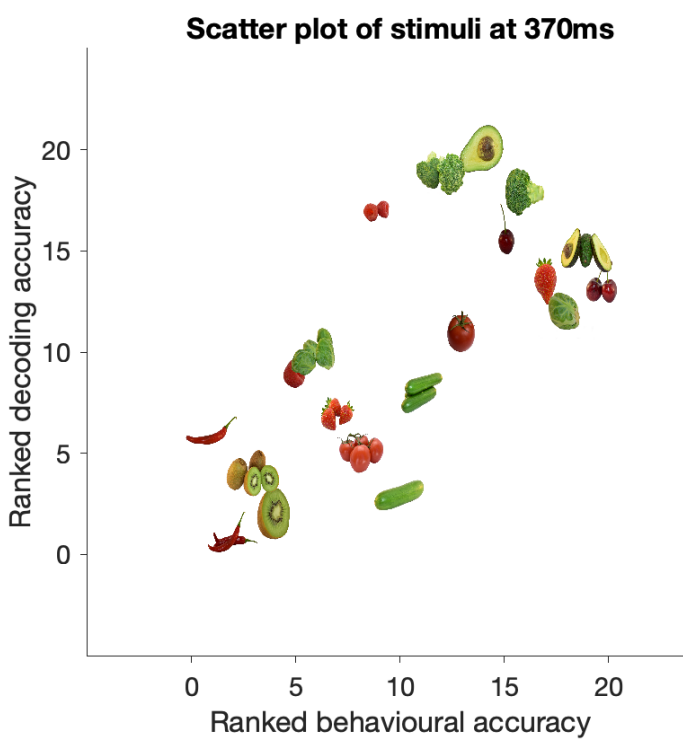

Figure 4: Panel A shows the correlation between the stimulus-wise behavioural accuracies in the independent colour categorisation task and the stimulus-wise MEG decoding accuracies over time. Panel B shows a scatterplot for the ranked behavioural accuracies and the ranked decoding accuracies for each stimulus at the peak timepoint of Panel A (370ms).

\section{Discussion}

323 In this study, we compared the temporal activation patterns of colour perception and implied colour to examine the interaction between perceptual processing and access to object representations. We applied MVPA to time-series MEG data and show that both real and implied colour can be decoded, with some caveats around implied colour decoding due to potential visual stimulus differences. Our key results indicate that real and implied colour processing share a sufficient degree of similarity to allow for cross-generalisation with a temporal shift. The activity pattern distinguishing colours was instantiated $\sim 55 \mathrm{~ms}$ later for implied colours than for real colour, highlighting that there are similarities between colour representations accessed via 'real' colour and via implied colour, but that there is a temporal asynchrony between these processes.

We interpret our cross-decoding results as evidence that the representation of implied 
335

336

337

in line with previous studies showing that the same brain regions are active when retrieving object representations from memory and perceiving those object features (for reviews see A. Martin, 2007; Patterson et al., 2007) For example, Vandenbroucke et al. (2014) and Bannert and Bartels (2013) showed that early visual cortex is involved when real and implied colour are processed. Using fMRI, Vandenbroucke et al. (2014) trained a classifier on data recorded while participants viewed red and green shapes, and then tested the classifier on data recorded while participants viewed line-drawings of colour-diagnostic objects filled with ambiguous colours between red and green. Behavioural results suggested participants were slightly more likely to respond 'red' to the ambiguous colour presented on a line drawing of a typically red object than a line drawing of a typically green object. In their fMRI data, the classifier categorised the colour consistent with what the participant perceived. That means the classifier categorised colours to be red when shown on objects that are typically red, and green for objects that are typically green, at above chance levels. They interpret these data as evidence for an influence of implied object colours on the creation of a subjective experience of colour. Consistent with this study, Bannert and Bartels (2013) trained a classifier to distinguish fMRI data from trials where four different colours were presented. They showed that the classifier can cross-generalise to grey-scale colour-diagnostic objects. Both fMRI studies highlight that there are similar activation patterns across voxels in the visual cortex for real and implied colour processing. Our results provide further evidence that object-colour knowledge and colour perception instantiate similar patterns, this time in the temporal domain.

There are several possible explanations for a temporal difference between accessing colour representations via real colour and implied colour. One possibility is that the time difference reflects greater individual variability in the temporal activation profile of implied colours in comparison to real colours. Implied colours may be accessed at slightly different timepoints for different people and thus the cross-decoding accuracy that is above chance for 
each participant only overlaps at a later timepoint. There are also more interesting potential explanations. First, it could be due to actual differences in neural processes. Colour representations accessed via colour perception are immediately available whereas implied colour activation presumably only happens once the object is processed to some higher level.

Thus, the delay could reflect differences between bottom-up and top-down access to colour representations. It might be, for example, that processing an object with a typical colour involves the activation of information about the object's implied colour which is fed-back to earlier visual areas to compare incoming information with stored object-knowledge. In comparison, the shapes used in the real colour trials are not associated with a typical colour and thus do not evoke such as signal. This is a plausible interpretation of the temporal delay and corresponds with earlier findings of early visual areas being involved in implied colour activation (Bannert \& Bartels, 2013; Vandenbroucke et al., 2014). Second, it is possible that the binding of colour and shape information happens later in the visual processing hierarchy compared to initial processing of separate features, and that the comparison of typical and perceived colour can therefore only happen later once shape-colour binding is complete. This view is consistent with results of a recent fMRI study, which showed that object-colour and object-shape activated from memory can be distinguished in areas associated with colour (V4) and shape (lateral occipital cortex, LOC) perception, respectively, but that the conjunction of

378 colour and shape can be decoded only later along the visual hierarchy (anterior temopral lobe,

379 ATL; Coutanche \& Thompson-Schill, 2014). Similarly, Seymour et al. (2015) showed that colour per se can be decoded in early visual areas but object surface colour (bound to form) can only be decoded in areas further along the ventral visual stream. These findings also correspond to patient work (Patterson et al., 2007) and previous transcranial magnetic

383 stimulation studies (Chiou, Sowman, Etchell, \& Rich, 2014) which point towards the ATL as the hub for object-knowledge (for a review see Lambon Ralph, Jefferies, Patterson, \& Rogers, 
385

386

387

388

389

390

391

392

393

394

396

397

398

399

400

401

402

403

404

405

406

407

408

409

2017). Besides the ATL, other brain areas along the processing stream such as the medial temporal lobe (e.g., Rey et al., 2018), and the parahippocampal cortex (e.g., C. B. Martin, Douglas, Newsome, Man, \& Barense, 2018) are also involved in retrieving long-term associations. Thus, it is possible that the temporal delay reflects the time it takes to activate these long-term colour associations. Finally, it could also be that the delay reflects the greater complexity of the grey-scale objects relative to the abstract shapes, hence binding the features may take slightly longer. From the data we cannot disentangle these interpretations. Our results clearly highlight, however, that there is a similar structure to the brain response to externally perceived and internally activated colour representations, and that time seems to be the key difference.

What is driving the successful decoding performance? For the real colour decoding, we used shapes that were identical across colour categories and used five different levels of stimulus luminance for each category that were perceptually matched. Therefore, the only distinguishing feature between the stimuli was colour. That means that for the real-colour decoding analysis and the cross-generalisation (i.e., training on shapes and testing on objects), we can rule out visual differences other than colour as a driving factor. Our results show that we can successfully decode real colour from $\sim 65 \mathrm{~ms}$ onwards. The within-implied colour decoding results show that implied colour is decodable at $\sim 190 \mathrm{~ms}$ after stimulus onset and then again a bit later at $\sim 270 \mathrm{~ms}$. This double-peak may occur because of variance between stimuli, such that accessing colour representations might be quicker for some images with stronger colour associations (for example) than others, or between participants in the speed with which they activate these representations. Alternatively, it may relate to differences in feedforward and feedback processes. For this within-implied colour classification analysis, visual differences could potentially contribute, as natural objects cannot be perfectly matched for the different conditions (i.e., red and green), unlike in our real colour condition. Previous studies 
410 have used line-drawings instead of photos of objects to reduce local low-level differences

411 between stimuli (e.g., Vandenbroucke et al., 2014). Line-drawings can reduce some of these

412 differences (e.g., local luminance differences due to texture) but also cannot completely rule

413 out any contribution of low-level effects (e.g., shapes). In addition, there is a considerable

414 trade-off between line-drawings in terms of similarity of the objects to real world objects which

415 can slow down recognition and implied colour effects (Olkkonen, Hansen, \& Gegenfurtner,

416 2008; Vurro, Ling, \& Hurlbert, 2013). We therefore used isoluminant, grey-scale photos of

417 objects and dealt with differences in low-level features (e.g., edges) by using an independent

418 exemplar cross-validation approach. We trained the classifier to distinguish typically red and

419 green objects using all objects except one typically-red and one typically-green object (each

420 with two exemplars, which were both left out). The classifier was then tested on the left-out

421 pair. We thereby considerably reduced the likelihood of the implied colour classification being

422 driven by low-level features as the classifier never trained and tested on the same objects. While

423 limiting the influence low-level features could have on the implied object colour decoding, it

424 is still possible that the results in this particular analysis are driven by object features other than

425 colour. To test this, we ran the same classification analysis on the output of a deep

426 convolutional neural network which showed that it is unlikely that low-level visual differences

427 account for all of the within-implied classification results (see supplementary material).

428 Crucially, however, visual differences are not a concern for the key cross-decoding analysis.

429 Here, we used identical red and green shapes in the training set, making low-level shape or

430 texture features a highly unlikely source of contribution to classifier performance and colour

431 hue being the primary predictor of category for the classifier (red vs green).

Our time-generalisation analysis shows that there are sufficient similarities in neural

433 representation when perceiving real colour and activating implied colour for cross-

434 generalisation. In addition, these results speak to the important aspect of temporal differences 
between colour evoked by external stimulation and internal activation. Activating conceptual knowledge of objects from memory is thought to involve a widespread network of brain areas involved in perception and action of different object features (A. Martin, 2007; Patterson et al., 2007). To access the implied colour of an object requires that the conceptual representation of that object is activated first. Using time-generalisation methods (King \& Dehaene, 2014), we

440 show here that in comparison to real colour perception, which can be decoded rapidly, accessing object-colour knowledge takes $\sim 55 \mathrm{~ms}$ longer. This is consistent with our behavioural data showing that real colour judgments are faster than implied colour judgments. The behavioural data do not, however, speak to the neural similarity between real and implied colour activation patterns, which are observed in the time-generalisation analyses. Our MEG results increase our existing knowledge of how real and implied colour are processed by showing that aspects of colour representations via external stimulation are also instantiated during internal activation, but with a delay. Applying MVPA to our MEG data allows us to capture the similarity of representations of real colour perception and implied colour activation, but also allow us to examine temporal differences, highlighting the value of this method for dissociating activation of memory of object features from perception of object features in the real world.

Our results highlight that the activation of implied colours can occur independent of a task that focuses on colour. Participants completed a target-detection task in which attending to colour was not a useful strategy. The targets were ambiguous in colour (e.g., a capsicum can be either red or green), and this avoided biasing participants towards deliberately thinking about the implied colour of the objects. Using a task that is irrelevant to the classifier performance allowed us to explore the involuntary activation of implied colours rather than the signals associated with perhaps actively imagining colours or retrieving colour names. Not 
accuracy in general (e.g., Brouwer \& Heeger, 2013; Jackson, Rich, Williams, \& Woolgar, 2017), but clearly supports previous studies showing that there is an involuntary activation of object-colour independent of task demands (Bannert \& Bartels, 2013; Vandenbroucke et al., 2014).

Overall, the decoding accuracies across our analyses are low but significantly above chance with conservative statistics. As outlined above, this is probably partially due to colour being irrelevant for the task. In addition, it is important to note that we did not use extensive pre-processing, meaning we ran our analyses on effectively raw data. We use our multivariate decoding analyses for interpretation (Hebart \& Baker, 2017)- if decoding is above chance, this means there is a signal that allows a categorical distinction between the conditions. Minimal pre-processing (e.g., no trial averaging, filtering, channel-selection, trial-selection) ensures that there is no potential influence of plurality of methods or specific pre-processing choices; it also means that the data overall are noisier which can result in relatively low decoding accuracies. However, it is crucial to note that low decoding accuracies does not necessarily mean that the effects are weak, as decoding accuracies are not effect sizes (cf. Hebart \& Baker, 2017). Here, we show with rigorous methodological controls and strict correction for multiple comparisons that there is significant cross-generalisation from real colour to implied colour. perception and implied colour activation (Bannert \& Bartels, 2013; Rich et al., 2006;

479 Vandenbroucke et al., 2014), but other studies implicate anterior temporal regions in object colour knowledge. For example, a transcranial magnetic stimulation study showed that the behavioural effects of implied colour knowledge on object recognition are disrupted when

482 stimulating the anterior temporal lobe (Chiou et al., 2014), complementing patient studies 483 suggesting this area holds conceptual object information (e.g., Lambon Ralph \& Patterson, 
485 low-level visual areas. Our study adds time as a novel aspect to this discussion by comparing

486 the temporal profiles of colour representations accessed via real colour perception and implied

487 colour activation.

488 In conclusion, our data show that there is a common representation of real and implied

489 colour but that this representation is accessed later when triggered by activating implied colour

490 than by perceiving real colour. This is in line with previous studies suggesting that the same

491 brain areas are involved in object-feature activation from memory and object-feature

492 perception. Our results highlight that applying MVPA to time-series MEG data is a valuable

493 approach to exploring the interaction between object-feature inputs and predictions or

494 representations based on prior knowledge. This opens multiple avenues for future studies

495 examining the dynamic interactions between perceptual processes and activation of prior 496 conceptual knowledge.

497 


\section{References}

499 Bannert, M. M., \& Bartels, A. (2013). Decoding the yellow of a gray banana. Current Biology, 23(22), 2268-2272.

Barsalou, L. W., Simmons, W. K., Barbey, A. K., \& Wilson, C. D. (2003). Grounding conceptual knowledge in modality-specific systems. Trends in Cognitive Sciences, $7(2), 84-91$.

Bartleson, C. J. (1960). Memory colors of familiar objects. JOSA, 50(1), 73-77.

Bramão, I., Faísca, L., Petersson, K. M., \& Reis, A. (2010). The influence of surface color information and color knowledge information in object recognition. The American Journal of Psychology, 123(4), 437-446.

Brouwer, G. J., \& Heeger, D. J. (2013). Categorical clustering of the neural representation of color. Journal of Neuroscience, 33(39), 15454-15465.

510 Carlson, T. A., Hogendoorn, H., Kanai, R., Mesik, J., \& Turret, J. (2011). High temporal resolution decoding of object position and category. Journal of Vision, 11(10), 9-9.

512 Chao, L. L., \& Martin, A. (1999). Cortical Regions Associated with Perceiving, Naming, and Knowing about Colors. Journal of Cognitive Neuroscience, 11(1), 25-35. https://doi.org/10.1162/089892999563229

515 Chiou, R., \& Rich, A. N. (2014). The role of conceptual knowledge in understanding synaesthesia: Evaluating contemporary findings from a "hub-and-spokes" perspective. Frontiers in Psychology, 5. https://doi.org/10.3389/fpsyg.2014.00105 burst stimulation to the left anterior temporal lobe untangles object representation and its canonical color. Journal of Cognitive Neuroscience, 26(5), 1066-1074.

521 Collins, J. A., \& Olson, I. R. (2014). Knowledge is power: How conceptual knowledge transforms visual cognition. Psychonomic Bulletin \& Review, 21(4), 843-860. 
523 Coutanche, M. N., \& Thompson-Schill, S. L. (2014). Creating concepts from converging features in human cortex. Cerebral Cortex, 25(9), 2584-2593.

de-Wit, L., Alexander, D., Ekroll, V., \& Wagemans, J. (2016). Is neuroimaging measuring information in the brain? Psychonomic Bulletin \& Review, 23(5), 1415-1428. https://doi.org/10.3758/s13423-016-1002-0

Engel, A. K., Fries, P., \& Singer, W. (2001). Dynamic predictions: oscillations and synchrony in top-down processing. Nature Reviews Neuroscience, 2(10), 704.

Firestone, C., \& Scholl, B. J. (2016). Cognition does not affect perception: Evaluating the evidence for" top-down" effects. Behavioral and Brain Sciences, 39.

Goldstone, R. L., de Leeuw, J. R., \& Landy, D. H. (2015). Fitting perception in and to cognition. Cognition, 135, 24-29.

Grootswagers, T., Cichy, R. M., \& Carlson, T. A. (2018). Finding decodable information that can be read out in behaviour. NeuroImage.

Grootswagers, T., Wardle, S. G., \& Carlson, T. A. (2017). Decoding Dynamic Brain Patterns from Evoked Responses: A Tutorial on Multivariate Pattern Analysis Applied to Time Series Neuroimaging Data. Journal of Cognitive Neuroscience, 29(4), 677-697.

Hansen, T., Olkkonen, M., Walter, S., \& Gegenfurtner, K. R. (2006). Memory modulates https://doi.org/10.1162/jocn_a_01068 color appearance. Nature Neuroscience, 9(11), 1367.

Hebart, M. N., \& Baker, C. I. (2017). Deconstructing multivariate decoding for the study of brain function. Neuroimage.

544 Hering, E. (1920). Grundzüge der Lehre vom Lichtsinn. Springer.

545 Jackson, J., Rich, A. N., Williams, M. A., \& Woolgar, A. (2017). Feature-selective attention in frontoparietal cortex: multivoxel codes adjust to prioritize task-relevant information. Journal of Cognitive Neuroscience, 29(2), 310-321. 
548 Kaiser, P. K. (1991). Flicker as a function of wavelength and heterochromatic ficker photometry. Limits of Vision, 171-190.

King, J. R., \& Dehaene, S. (2014). Characterizing the dynamics of mental representations: the temporal generalization method. Trends in Cognitive Sciences, 18(4), 203-210. https://doi.org/10.1016/j.tics.2014.01.002

Kleiner, M., Brainard, D., Pelli, D., Ingling, A., Murray, R., Broussard, C., \& others. (2007). What's new in Psychtoolbox-3. Perception, 36(14), 1.

Lambon Ralph, M. A., Jefferies, E., Patterson, K., \& Rogers, T. T. (2017). The neural and computational bases of semantic cognition. Nature Reviews Neuroscience, 18(1), 42.

Lambon Ralph, M. A., \& Patterson, K. (2008). Generalization and differentiation in semantic memory: insights from semantic dementia. Annals of the New York Academy of

Maris, E., \& Oostenveld, R. (2007). Nonparametric statistical testing of EEG-and MEG-data. Journal of Neuroscience Methods, 164(1), 177-190.

Martin, A. (2007). The representation of object concepts in the brain. Annu. Rev. Psychol., $58,25-45$.

Martin, A., Haxby, J. V., Lalonde, F. M., Wiggs, C. L., \& Ungerleider, L. G. (1995). Discrete ventral visual stream. Elife, 7, e31873. objects: Effects of object shape, texture, and illumination changes. Journal of Vision, 8(5), 13-13. 
573 Oostenveld, R., Fries, P., Maris, E., \& Schoffelen, J.-M. (2011). FieldTrip: open source software for advanced analysis of MEG, EEG, and invasive electrophysiological data. Computational Intelligence and Neuroscience, 2011, 1.

Oosterhof, N. N., Connolly, A. C., \& Haxby, J. V. (2016). CoSMoMVPA: multi-modal multivariate pattern analysis of neuroimaging data in Matlab/GNU Octave. Frontiers in Neuroinformatics, 10. Retrieved from https://www .ncbi.nlm.nih.gov/pmc/articles/PMC4956688/

Patterson, K., Nestor, P. J., \& Rogers, T. T. (2007). Where do you know what you know? The representation of semantic knowledge in the human brain. Nature Reviews Neuroscience, 8(12), 976.

Pelli, D. G. (1997). The VideoToolbox software for visual psychophysics: Transforming numbers into movies. Spatial Vision, 10(4), 437-442.

Rey, H. G., De Falco, E., Ison, M. J., Valentin, A., Alarcon, G., Selway, R., ... Quiroga, R. Q. (2018). Encoding of long-term associations through neural unitization in the human medial temporal lobe. Nature Communications, 9(1), 4372.

Rich, A. N., Williams, M. A., Puce, A., Syngeniotis, A., Howard, M. A., McGlone, F., \& Mattingley, J. B. (2006). Neural correlates of imagined and synaesthetic colours. Neuropsychologia, 44(14), 2918-2925.

Seymour, K. J., Williams, M. A., \& Rich, A. N. (2015). The representation of color across the human visual cortex: distinguishing chromatic signals contributing to object form versus surface color. Cerebral Cortex, 26(5), 1997-2005.

594 Simmons, W. K., Ramjee, V., Beauchamp, M. S., McRae, K., Martin, A., \& Barsalou, L. W. (2007). A common neural substrate for perceiving and knowing about color. Neuropsychologia, 45(12), 2802-2810. 
597 Smith, S. M., \& Nichols, T. E. (2009). Threshold-free cluster enhancement: addressing problems of smoothing, threshold dependence and localisation in cluster inference. Neuroimage, 44(1), 83-98.

600 van Driel, J., Olivers, C. N., \& Fahrenfort, J. J. (2019). High-pass filtering artifacts in 601 multivariate classification of neural time series data. BioRxiv, 530220.

602 Vandenbroucke, A. R., Fahrenfort, J. J., Meuwese, J. D. I., Scholte, H. S., \& Lamme, V. A. 603 F. (2014). Prior knowledge about objects determines neural color representation in 604 human visual cortex. Cerebral Cortex, 26(4), 1401-1408.

605 Vurro, M., Ling, Y., \& Hurlbert, A. C. (2013). Memory color of natural familiar objects:

606 Effects of surface texture and 3-D shape. Journal of Vision, 13(7), 20-20.

607 Williams, M. A., Dang, S., \& Kanwisher, N. G. (2007). Only some spatial patterns of fMRI 608 response are read out in task performance. Nature Neuroscience, 10(6), 685.

609 Witzel, C. (2016). An easy way to show memory color effects. I-Perception, 7(5), $610 \quad 2041669516663751$. 